\title{
Correction to: IncRNA MEG3, Acting as a ceRNA, Modulates RPE Differentiation Through the miR-7-5p/Pax6 Axis
}

\author{
Hong-Jing Sun ${ }^{1} \cdot$ Fang-Fang Zhang ${ }^{2} \cdot$ Qing Xiao $^{1} \cdot$ Jia Xu$^{1} \cdot \mathrm{Li}-$ Jin Zhu ${ }^{2}$
}

Published online: 1 June 2021

๑) Springer Science+Business Media, LLC, part of Springer Nature 2021

\section{Correction to: Biochemical Genetics https://doi.org/10.1007/s10528-021-10072-9}

The original version of this article unfortunately contained a mistake. In the original version under the heading 'Introduction', the last paragraph text read as:

In this study, we showed that MEG3 was downregulated in REP-choroid samples of AMD patients. We also demonstrated that downregulated MEG3 enhanced RPE differentiation by regulating the miR-7-5p/Pax6 axis. Considering the crucial role of RPE dedifferentiation in the AMD pathological process, lncRNA MEG3 may be a promising target for the treatment of AMD.

Please find below the corrected text:

In this study, we demonstrated that downregulated MEG3 enhanced RPE differentiation by regulating the miR-7-5p/Pax6 axis. Considering the crucial role of RPE dedifferentiation in the AMD pathological process, lncRNA MEG3 may be a promising target for the treatment of AMD.

The original article has been corrected.

Publisher's Note Springer Nature remains neutral with regard to jurisdictional claims in published maps and institutional affiliations.

The original article can be found online at https://doi.org/10.1007/s10528-021-10072-9.

Li-Jin Zhu

lijinzhu@hmc.edu.cn

1 Department of Ophthalmology, The Second Affiliated Hospital, Zhejiang University School of Medicine, Hangzhou 310009, Zhejiang, People's Republic of China

2 School of Public Health, Hangzhou Medical College, No. 182, Tianmushan Road, Hangzhou 310013, Zhejiang, People's Republic of China 